\title{
Heart Disease Diagnosis Using Image Processing By Cuckoo Algorithm before Surgery
}

\author{
Pouya Derakhshan-Barjoei ${ }^{1 *}$, Mojdeh Bahadorzadeh ${ }^{2}$ and Elham Foroozandeh ${ }^{3}$ \\ ${ }^{1}$ Department of Telecommunication Engineering, Iran \\ ${ }^{2}$ Department of General Surgery, Iran \\ ${ }^{3}$ Department of Psychology, Iran
}

*Corresponding author: Pouya Derakhshan-Barjoei, Department of Telecommunication Engineering, Naein Branch, Naein, Iran

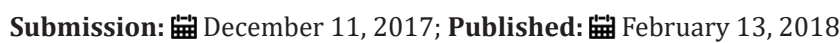

\begin{abstract}
The study of heart disease is important because of urgency of diagnosis. In the meantime, the discussion of image processing and diagnosis is important in medical angiography images, a quick diagnosis before surgery. All of these are for preventing delayin surgery. Therefore, in this study, diagnostic methods using cuckoo algorithm and medical engineering optimization methods is proposed. we presented an optimal bio inspired diagnostic model. The Coronary angiography using dedicated X-ray imaging systems has long been the preferred modality for diagnosis and treatment of Coronary Artery Disease (CAD) and its concomitants. Experimental results on several test images of patients with various techniques are presented. The results of our fast bio inspired diagnostic model performed well. Using bio-inspired algorithm as cuckoo optimization in order to edge detection including noise reduction has been done. the angiogram interpretation has been discussed with medical consultant.
\end{abstract}

\section{Introduction}

True imaging and diagnosis can be very helpful to the surgeon, and if the imaging is disturbed, it may have a bad impact on the individual. Angiographic and interventional radiologic techniques are also performed with injected contrast. In this instance, catheter insertion or needle placement is performed under fluoroscopic guidance, so that a particular vessel or organ can be see and, in some instances, repaired via the catheter. A classic example is angioplasty, for which a catheter is threaded into an obstructed vessel. An attached balloon is inflated to increase the opening of the vessel thus improving blood supply to the tissues. This procedure is commonly performed on vessels in the heart, abdomen, and legs. As one might expect, these interventional techniques are complex procedures, involving teams of nurses, doctors, and technologists working together. The great advantage is that angioplasty and other image-guided interventional procedures replace the need for (and risks of) surgery and general anesthesia for many patients. Even for other surgeries, the doctor may need to have a better picture and diagnosis with higher accuracy. The interventional service offers more specific information and advice for patients undergoing these procedures [1-4]. Apart from rare congenital anomalies (birth defects), coronary artery disease is usually a degenerative disease, uncommon as a clinical problem before the age of 30 years and common by the age of 60 years. One in four people will have a heart attack [5]. cardiac blockages are detected by means of Canny edge detector and Watershed image processing algorithms implemented on FPGA [6].

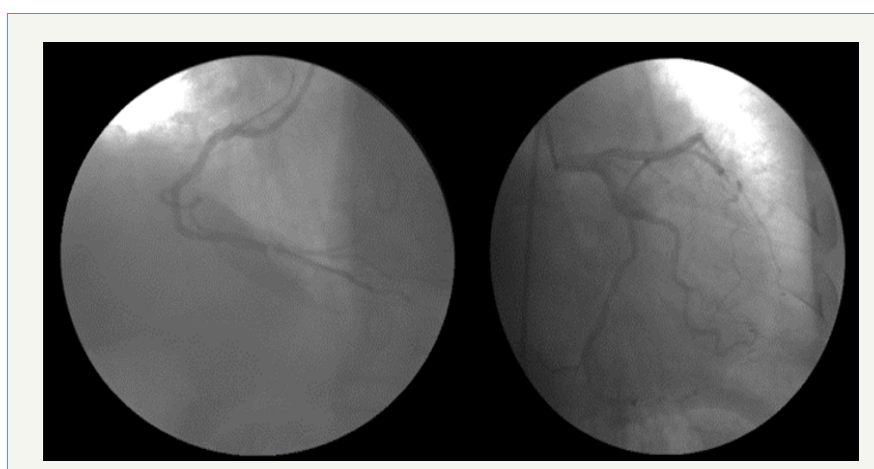

Figure 1: Frames of Coronary angiology.

Conventional X-ray coronary angiograms were performed as part of a clinically indicated assessment with an integrated digital cardiac catheter imaging system. Atherosclerosis is a multifactorial chronic inflammatory, degenerative process of large-and mediumsized arteries characterized pathologically by the presence of atherosclerotic plaque. The clinical signs and symptoms of atherosclerosis are generally summarized under the term cardiovascular disease. Shown in Figure 1, typical cardiovascular disease is coronary artery disease (angina and myocardial infarction), cerebrovascular disease (stroke) and peripheral arterial occlusive 
disease. Cardio-vascular diseases are the leading cause of mortality in developed countries [7-10]. The best pre processing is a nonlinear "variant" filtering, where each pixel is replaced by the average of the $3 \times 3$ neighborhood having the smallest variance [11]. In this method, the patient's photos were first considered and then, with the optimal processing method we proposed, the results were estimated and diagnosed and consulted by the surgeon's opinion.

\section{Cuckoo Algorithm for Image Processing}

This algorithm is one of the newest and most powerful evolutionary optimization methods ever introduced. Cuckoo's algorithm is inspired by the bird's way of life called Cuckoo, developed by Xin-she Yang and Suash Deb in 2009 [12-14]. Cuckoo algorithm is based on the life of the species of cuckoo. It was inspired by the obligate brood parasitism of some cuckoo species by laying their eggs in the nests of other host birds. Some host birds can engage direct conflict with the intruding cuckoos. This algorithm is based on three idealized rules: Each cuckoo lays one egg at a time, and dumps its egg in a randomly chosen nest; The best nests with high quality of eggs will carry over to the next generation; The number of available hosts nests is fixed, and the egg laid by a cuckoo is discovered by the host bird with a determined probability. Discovering operate on some set of worst nests, and discovered solutions dumped from farther calculations (Table 1).

Table 1: Cuckoo parameters.

\begin{tabular}{|c|c|}
\hline Cuckoo population & 15 \\
\hline Minimum number of eggs & 4 \\
\hline Maximum number of eggs & 8 \\
\hline Alive cuckoo & 100 \\
\hline Population variance & $08-10$ \\
\hline
\end{tabular}

The concept of mutual information was introduced for the first time to detect the relationship between these features of a data set. The speed and accuracy of the algorithm behaved better than our previous method [15]. Our proposed algorithm, involves the following steps.

A. Step 1: Read the given angiogram image, and convert it into amatrix form, where each pixel value is in the range of gray level from 0-255.

B. Step 2: Apply filtering and noise reduction method.

C. Step 3: Taking Histogram sample and equalization.

D. Step 5: Repeat process.

E. Step 6: Detect the edges of the angiogram using Cuckoo algorithm to optimize the edge

F. Step 7: Repeat for the best result.

G. Step 8: Continue to optimize and update the parameters of Cuckoo algorithm.

H. Step 9: Repeat.
The output of the various edge detected angiogram images to obtain the edges of the blood vessel is shown by the histogram its histogram equalized image. The results show that the edges of the angiogram blood vessel so detected using the proposed algorithm is more efficient as compared to the canny edge detection algorithm. In Figure 2, the result of experience on one cardiac frame is showed, as our method shows, using optimal method is preferred and yields improved results. Now we are going to develop a method and algorithm to detect the disease of heart clearly and help the physician to fast preventing and good health care.
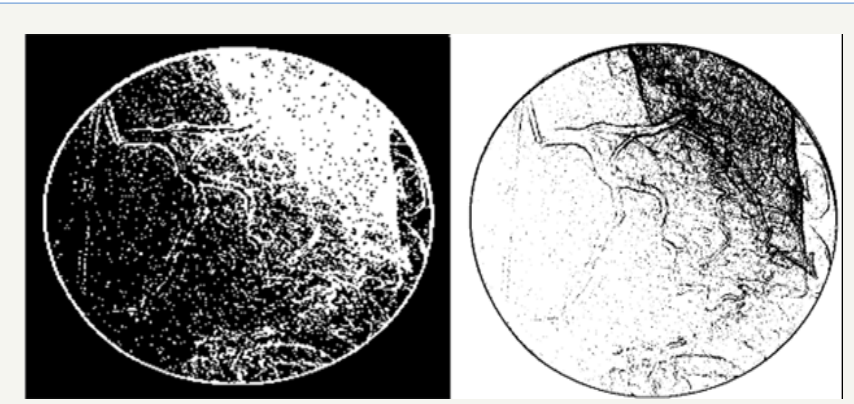

Figure 2: picture of one frame after: a) edge detection, b) negative edge detection.

\section{Conclusion}

Our bio-inspired cuckoo optimization algorithm shows the improvement of the images. It was very clear and accepted for tracking by physician as well. The bio-inspired technique showed the speed and accuracy of the algorithm better than related to our previous method. It is also very effective in detecting and demonstrating the effect of vascular or artery congestion in surgery. In this method, first, the patient's pictures were taken immediately and then, with the optimal processing method we proposed, the results of estimation and diagnosis were carried out by portable devices. Using this cuckoo inspired algorithm and using the imagenoise reduction filter, and fixing the image blurring, improved image quality and recognition.

\section{References}

1. H Liu, J Xu, Fajardo LL, Yin S, Yu FTS, et al. (1999) Optical processing architechture and its potential application for digital and analog radiography. Med phys 26: 648-652.

2. Chilcote WA, Modic MT, pavlicek WA, Little JR, Furlan AJ, et al. (2000) Digital subtraction angiography of the carotid arteries: a compatative study in 100 paitients. radiology 139(2): 287-295.

3. Ray TY (2002) the coronary Angiogram and its seminal contributions to cardiovascular mediine over five decades. circulation 106(6): 752-756.

4. Derakhshan P, Behrad BA (2007) Angiography Image Processing and Heart Disease Detection Using Gaussian Interpolation Method. ICEI, Malaysia.

5. Earls JP, Woodard PK, Abbara S, Akers SR, Araoz PA, et al. (2014) ACR appropriateness criteria asymptomatic patient at risk for coronary artery disease. J Am Coll Radiol 11(1): 12-19.

6. Mudigoudar SB, Rasheed AI (2016) Design and implementation of image processing algorithms for cardiac blockage detection on FPGA. 2016 IEEE Annual India Conference (INDICON), pp. 1-5. 
7. Jain AK (1989) Fundamentals of digital image processing. Prentice-Hall, USA.

8. Shaw CG, Ergun DL, Myerowitz PD (1982) A technique of scatter and glare correction for video densitometric studies in digital sub-traction video angiography. Radiology 142(1): 209-213.

9. Weinhaus AJ, Roberts KP (2005) Anatomy of the Human Heart. In: Iaizzo PA [Ed.] Handbook of Cardiac Anatomy, Physiology, and Devices. Humana Press, USA, pp. 51-79.

10. Derakhshan P, Bahadorzadeh BM (2017) An Intelligent Method for Diagnosis of Breast Cancer. Int J of Innovative Res in Elec and Comm 4(2): $6-12$.

11. Romary D, Lerallut JF, Fontenier G (1985) Application of image processing techniques to gamma-angiography. Comput Biomed Res 18(5): 488495.

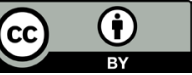

reative Commons Attribution 4.0

International License

For possible submission use the below is the URL
12. Yang XS, Deb S (2009) Cuckoo search via Lévy flights. World Congress on Nature \& Biologically Inspired Computing, IEEE Publications, India, pp. 210-214.

13. Payne RB, Sorenson MD, Klitz K (2005) The Cuckoos. Oxford University Press, India.

14. Rajabioun R (2011) Cuckoo Optimization Algorithm. Applied Soft Computing Journal 11(8): 5508-5518.

15. Derakhshan P, Bahadorzadeh M (2012) Enhancement in Medical Image Processing for Breast Calcifications and Tumor Detection. Research Journal of Applied Sciences, Engineering and Technology 4(12): 16961700.

\section{Your subsequent submission with Crimson Publishers} will attain the below benefits

- High-level peer review and editorial services

- Freely accessible online immediately upon publication

- Authors retain the copyright to their work

- Licensing it under a Creative Commons license

- Visibility through different online platforms

- Global attainment for your research

- Article availability in different formats (Pdf, E-pub, Full Text)

- Endless customer service

- Reasonable Membership services

- Reprints availability upon request

- One step article tracking system 